\title{
Absolute quantitative total-body small-animal SPECT with focusing pinholes
}

\author{
Chao Wu • Frans van der Have • \\ Brendan Vastenhouw • Rudi A. J. O. Dierckx • \\ Anne M. J. Paans • Freek J. Beekman
}

Received: 23 February 2010 / Accepted: 3 June 2010 / Published online: 25 June 2010

(C) The Author(s) 2010. This article is published with open access at Springerlink.com

\begin{abstract}
Purpose In pinhole SPECT, attenuation of the photon flux on trajectories between source and pinholes affects quantitative accuracy of reconstructed images. Previously we introduced iterative methods that compensate for image degrading effects of detector and pinhole blurring, pinhole sensitivity and scatter for multi-pinhole SPECT. The aim of this paper is (1) to investigate the accuracy of the Chang algorithm in rodents and (2) to present a practical Changbased method using body outline contours obtained with optical cameras.

Methods Here we develop and experimentally validate a practical method for attenuation correction based on a
\end{abstract}

C. Wu $(\bowtie) \cdot$ R. A. J. O. Dierckx · A. M. J. Paans

Department of Nuclear Medicine and Molecular Imaging,

University Medical Center Groningen, University of Groningen,

Hanzeplein 1, P.O. Box 30001, 9700 RB Groningen,

The Netherlands

e-mail:wuc@ngmb.umcg.nl

C. $\mathrm{Wu}$

Graduate School for Drug Exploration,

University Medical Center Groningen, University of Groningen,

Groningen, The Netherlands

C. Wu $\cdot$ F. van der Have $\cdot$ B. Vastenhouw $\cdot$ F. J. Beekman

Image Sciences Institute and Rudolf Magnus Institute,

University Medical Center Utrecht,

Utrecht, The Netherlands

F. van der Have $\cdot$ B. Vastenhouw $\cdot$ F. J. Beekman

MILabs B.V.,

Utrecht, The Netherlands

F. van der Have $\cdot$ B. Vastenhouw $\cdot$ F. J. Beekman

Department R3, Section Radiation, Detection \& Medical Imaging,

Delft University of Technology,

Delft, The Netherlands
Chang first-order method. This approach has the advantage that it is employed after, and therefore independently from, iterative reconstruction. Therefore, no new system matrix has to be calculated for each specific animal. Experiments with phantoms and animals were performed with a highresolution focusing multi-pinhole SPECT system (USPECT-II, MILabs, The Netherlands). This SPECT system provides three additional optical camera images of the animal for each SPECT scan from which the animal contour can be estimated.

Results Phantom experiments demonstrated that an average quantification error of $-18.7 \%$ was reduced to $-1.7 \%$ when both window-based scatter correction and Chang correction based on the body outline from optical images were applied. Without scatter and attenuation correction, quantification errors in a sacrificed rat containing sources with known activity ranged from -23.6 to $-9.3 \%$. These errors were reduced to values between -6.3 and $+4.3 \%$ (with an average magnitude of $2.1 \%$ ) after applying scatter and Chang attenuation correction.

Conclusion We conclude that the modified Chang correction based on body contour combined with window-based scatter correction is a practical method for obtaining small-animal SPECT images with high quantitative accuracy.

Keywords Quantification - Quantitative imaging · Small-animal imaging $\cdot$ SPECT

\section{Introduction}

Pinhole SPECT provides high-resolution images of small animals that can be used to quantitatively study the in vivo distribution of a new tracer or drug, for example to determine whether and how molecules reach the target area 
or what receptors are available in the animal. SPECT can also be employed for function or lesion detection with the help of a wide range of available radiolabelled molecules. In many cases far fewer animals need to be sacrificed in SPECT studies than in post-mortem tissue distribution studies, because SPECT allows for dynamic imaging and longitudinal studies and provides 3-D images with slices that are perfectly aligned to each other.

Several dedicated small-animal SPECT systems have been proposed. Most of them (e.g. [1-9]) employ (multi-) pinhole collimation instead of parallel-hole collimation that is used clinically, taking advantage of the magnification of pinholes to improve resolution [1, 10]. Small-animal SPECT images are typically much less degraded by photon scattering and absorption than clinical SPECT images because of smaller body dimensions. Nevertheless, the degradation in small-animal SPECT images is not negligible. For example in the centre of a rat-sized cylinder of water, photon attenuation can reduce the measured concentration of activity up to $25 \%$ when imaging with ${ }^{99 \mathrm{~m}} \mathrm{Tc}$ [11]. For clinical SPECT devices several attenuation correction [12-15] and scatter correction methods [14-20] have been developed. Several of these systems are now commercially available and their accuracy has been improved by the availability of integrated SPECT/CT devices [21, 22]. There are few publications about quantitative small-animal SPECT however (e.g. [11, 23, 24]). Recently, Vanhove et al. [25] presented their studies with an average error of $-7.9 \pm 10.4 \%$ between the activity concentrations measured on their scatter- and attenuation-corrected pinhole SPECT mouse images and in a dose calibrator. They used microCT imaging for producing attenuation maps, which has the advantage that non-uniformities can be taken into account but at the cost of increased dose to animals and need for additional hardware. Furthermore, the attenuation correction was incorporated in the iterative reconstruction process, which in some reconstruction algorithms may cause problems since they require a new system matrix for each subject to be imaged.

Post-reconstruction attenuation correction algorithms, such as the Chang method, had been proposed decades ago [26]. Their big advantage is that they do not need new system matrices. The first-order correction provided by the Chang algorithm is often not accurate enough for clinical use because effects of attenuation in patients are very strong. The aim of this paper is (1) to investigate the accuracy of the Chang algorithm in rodents and (2) to present a practical Chang-based method using body outline contours obtained with optical cameras. The method was tested for the case of focusing pinhole SPECT $[5,27]$ and in combination with correction of other effects such as scatter and distancedependent collimator blurring and sensitivity.

\section{Materials and methods}

U-SPECT-II: a focusing pinhole small-animal SPECT system

U-SPECT-II [9] is a stationary focusing multi-pinhole SPECT system for small-animal organ and total-body imaging studies. Exchangeable cylindrical collimators containing 75 focusing pinholes can be mounted in the centre and are surrounded by three NaI gamma cameras. Optical photos are acquired by three integrated optical cameras for volume of interest (VOI) selection before SPECT acquisition (Fig. 1). With an XYZ stage, an animal can be moved inside the collimator during imaging in order to also enable obtaining total-body images.

The U-SPECT-II system can reach sub-half-millimetre resolution. With ${ }^{99 \mathrm{~m}} \mathrm{Tc}$, the image resolution is better than $0.35 \mathrm{~mm}$ in any part of a mouse-sized object or better than $0.8 \mathrm{~mm}$ in any part of a rat-sized object [9]. With the scanner hardware and acquisition software, the information, including scintillation time, position and photon energy, etc., of every scintillation event is recorded in list mode [9]. This offers great flexibility for image reconstruction, such as implementing decay and spectrum- (e.g. window-) based scatter correction. More detailed descriptions, evaluations and examples of applications of the U-SPECT systems were given in $[5,9,28,29]$.

\section{Image reconstruction}

The scanning focus method (SFM) described in [30] was used for acquisition. With the SFM, a total-body scan can be carried out with a sequence of bed positions, and its image can be reconstructed with a single series of iterations. The system matrix used for computing re-projections and backprojections during iterative reconstruction with pixel-based ordered subset expectation maximization (POSEM [31]) is derived from point spread function (PSF) measurements [32]. Within these PSF-based matrices, the effects of the detector blurring, pinhole blurring and pinhole sensitivity are compensated.

\section{Calibration factor}

We define the calibration factor to be the ratio of the activity concentration to the voxel value in reconstructed SPECT images. Since the various distance-dependent pinhole sensitivities are already modelled in the system matrix and subsequently compensated in the reconstruction process [32], the calibration factor should be theoretically homogeneous throughout all voxels of reconstructions if attenuation and scatter can be neglected. It means that the calibration factor is a global scaling factor; thus we can 
Fig. 1 U-SPECT-II. a Overview of system. b Three integrated optical cameras. c User interface, showing optical photos for VOI selection

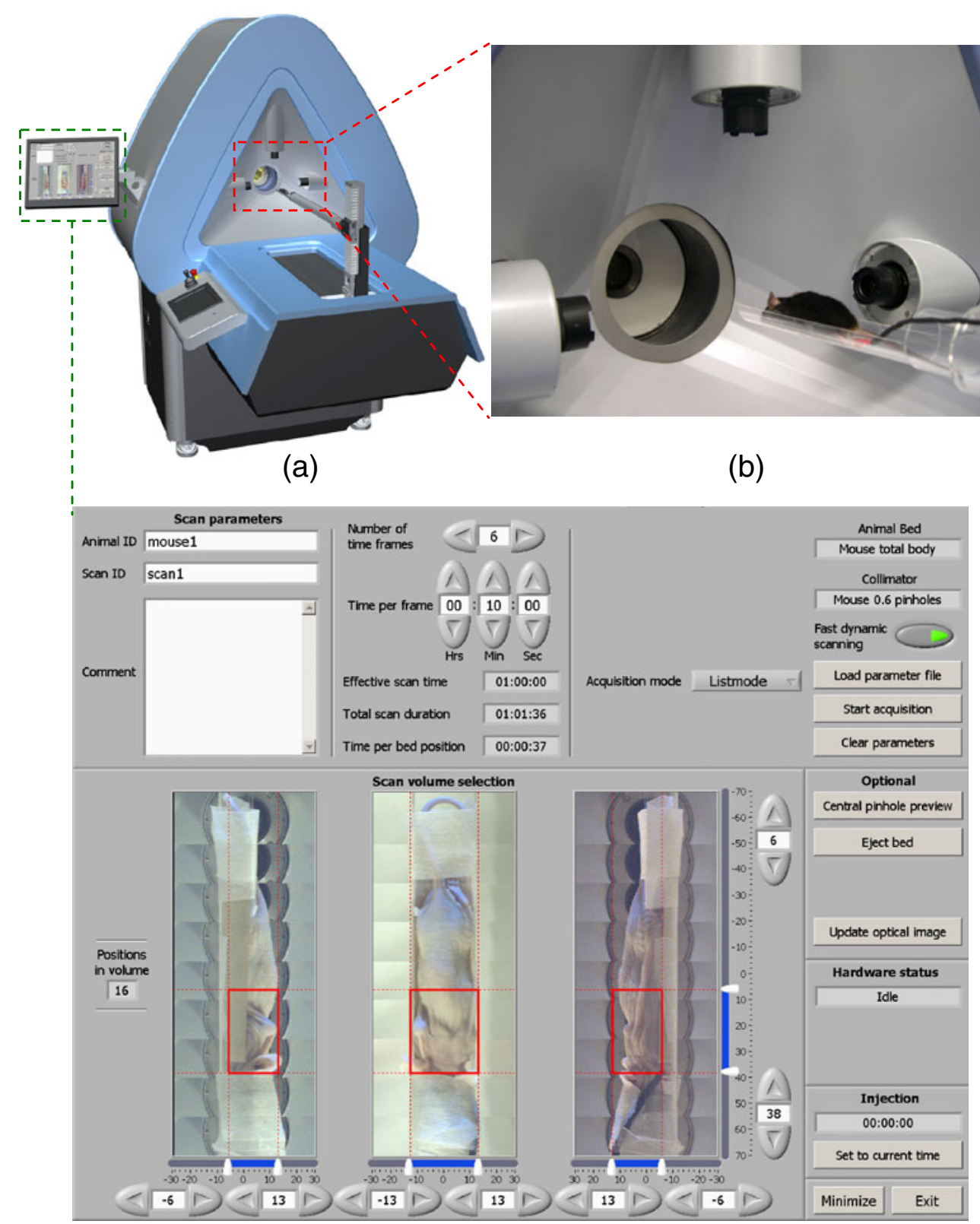

(c) obtain the factor by measuring and reconstructing a point source that is almost attenuation free.

We prepared a ${ }^{99 \mathrm{~m}} \mathrm{Tc}$ point source. The activity of the source was $69.0 \mathrm{MBq}$ measured in a VIK-202 dose calibrator. The calibration scan lasted for $200 \mathrm{~min}$, and then a volume of $10 \times 10 \times 10 \mathrm{~mm}^{3}$ containing the point source in the centre was reconstructed by running 6 POSEM iterations with 16 subsets. Decay effect was compensated during the reconstruction.

The calibration factor, $C F$, was given by

$C F=\frac{A}{V \cdot \sum R}$, where $A$ is the activity of the point source measured in the dose calibrator, $\sum R$ is the summation of voxel values all over the image and $V$ is the volume of a voxel. If $A$ has a unit of $\mathrm{MBq}, V$ is expressed in millilitres, and voxel value $R$ is considered to be dimensionless, then the $C F$ has a unit of $\mathrm{MBq} / \mathrm{ml}$.

For scatter- and attenuation-free acquisitions and reconstructions, after scaling by the $C F$, the voxel values directly represent the activity concentration in those voxels' local regions. However, in practice, scatter correction and attenuation correction should be carried out apart from the global scaling of voxel values. 


\section{Scatter correction}

In the U-SPECT-II system, scatter correction is integrated into the reconstruction step. Since data are acquired in list mode, scatter and photopeak windows can be set after acquisition. We employed the triple energy window (TEW) technique [33] in both phantom and animal experiments for this paper. A photopeak window $(140 \mathrm{keV}, 20 \%$ width) was used. Two background windows (centred at $117 \mathrm{keV}, 10 \%$ width and centred at $163 \mathrm{keV}, 7 \%$ width, respectively) were set adjacent to the photopeak for estimating the number of scattered photons of which the energies ranged inside the photopeak window. The scatter images were scaled by the ratio of the window widths, and added to the estimated scatter-free projections in the denominator of the POSEM formula along the lines proposed in Bowsher et al. [34]. In this way the contributions of scattered photons in projections were taken into account in order to eliminate their detriment to the images as much as possible.

This scatter correction scheme was also performed during the reconstruction of the point source used for obtaining the calibration factor. That scatter needs to be taken into account here is because although probability of scattering inside the point source is quite small, the amount of scattering by the imaging system, especially by the collimator, is not negligible $[35,36]$. In a reconstruction of the point source without scatter correction, we found that the calibration factor is $4.35 \%$ smaller than the one with scatter correction.

\section{Attenuation correction}

The Chang method [26] is a very practical first-order attenuation correction algorithm. It can be implemented as a post-reconstruction processing method, so that no new system matrix is needed. In recent clinical SPECT software, it has often been replaced by more accurate iterative attenuation correction. However, due to the small amount of attenuation in rodents, the Chang algorithm could be sufficient. If the over- and/or undercorrection problems of the Chang algorithm can be ignored, the attenuation correction process may benefit from the method's simplicity and high computation speed.

The consequence of attenuation is a reduction in the number of gamma photons which can arrive directly at the detectors, caused by photon scattering and absorption. The amount of attenuation depends on the photon energy, medium properties and the travelling distance of gamma photons in the medium. The transmitted fraction $(T F)$ is therefore represented as

$T F_{L}=\exp \left(-\int_{L} \mu(l) d l\right)$, where $L$ denotes the travelling path of a gamma photon inside the attenuation medium, and $\mu$ is the attenuation coefficient. The number of counts detected in that path is then reduced to

$N=N_{0} T F_{L}$,

where $N_{0}$ represents the number of counts detected without attenuation.

Chang [26] provided an approximation here: the $T F$ of a voxel over all possible projection paths is the average of all $T F_{L} \mathrm{~S}$, or

$T F=\frac{1}{M} \sum_{m=1}^{M} \exp \left(-\int_{L_{m}} \mu(l) d l\right)$,

where $M$ is the total number of projections taken in acquisition. In small-animal SPECT, a small $M$ could be sufficient due to the small amount of attenuation. To estimate a sufficiently large $M$ for a rat-sized object, we calculated the $T F$ s with different $M$ on a single slice with an attenuation coefficient of $0.151 \mathrm{~cm}^{-1}$ ( $=\mu$ of $140 \mathrm{keV}$ photon travelling in water) inside an area of an ellipse with its major and minor axes equal to 4 and $2 \mathrm{~cm}$, respectively. Then we took the $T F \mathrm{~s}$ of the voxels calculated with $M=1,024$ as reference data and inspected the normalized root mean square deviation (NRMSD) when $M$ is smaller. The results are listed in Table 1 . We found that by increasing $M$ above 32 gamma ray directions, the NRMSDs are below $0.2 \%$. Therefore, we considered $M=32$ as sufficiently large for a rat-sized object and applied it in our studies.

An attenuation map was needed to determine the attenuation coefficient $\mu$ in different locations of the image volume. In order to simplify the process, we considered the $\mu$ to be homogeneous and equal to $0.151 \mathrm{~cm}^{-1}$ ( $=\mu$ of $140 \mathrm{keV}$ photon travelling in water) inside the regions of the objects scanned. In this scheme, only the contour information of the objects was required.

An application program was developed for defining top view and side view 2-D contours of animals on the optical photos that standardly are taken before U-SPECT acquisition, e.g. for the purpose of VOI selection and activity localization. As shown in Fig. 2a, the three optical photos are displayed on the graphical user interface of the software, with a closed Bézier spline curve lying on top of each. The curves are initialized with standard shapes and can be deformed to fit animal outlines by dragging several anchor points. After proper 2-D contours were made, the software measured the width $p$ and height $q$ of the animal on the top view and side view contours, respectively, in each position of those transverse slices (Fig. 2b). Then it created an ellipse of which the horizontal and vertical axes 
Table 1 NRMSD of $T F$ s on an elliptic cross section between different $M$ and $M=1,024$

\begin{tabular}{llllllllll}
\hline$M$ & 4 & 8 & 16 & 32 & 64 & 128 & 256 & 512 & 1,024 \\
\hline NRMSD(\%) & 10.63 & 1.31 & 0.29 & 0.14 & 0.08 & 0.04 & 0.02 & 0.00 & 0 \\
\hline
\end{tabular}

were equal to $p$ and $q$, respectively, determined by the following equation:

$\frac{x^{2}}{p^{2}}+\frac{y^{2}}{q^{2}}=4$.

All those ordered ellipses were stacked together to form a 3-D contour of the object (Fig. 2c).

\section{Quantification}

With this 3-D contour and some extra information, such as voxel size and attenuation coefficient $\mu$, the software was able to compute the $T F$ of every voxel:

$T F=\frac{1}{32} \sum_{m=1}^{32} \exp \left(-\mu L_{m}\right)$.
It is important to compute $T F$ s for not only the voxels inside a 3-D contour, but also the ones outside. A source can exist outside an attenuation medium, e.g. due to an overly tight body contour, and gamma rays emitted by that source and penetrating the medium will still be attenuated, so that the $T F \mathrm{~s}$ for that source outside the 3-D contour should not be simply set to 1 . Another advantage is that it makes the $T F$ change continuously across the border of the contour, which reduces the error brought in by an inaccurate contour.

Finally we computed the activity concentration $A C$ at the location of every voxel of the reconstructed image, with the equation

$A C=\frac{R \cdot C F}{T F}$,

in which $R$ was the scatter-corrected voxel value.
Fig. 2 Generating a 3-D contour. a Graphical user interface. b 2-D contours. c A mesh plot of 3-D contours based on a stack of ellipses

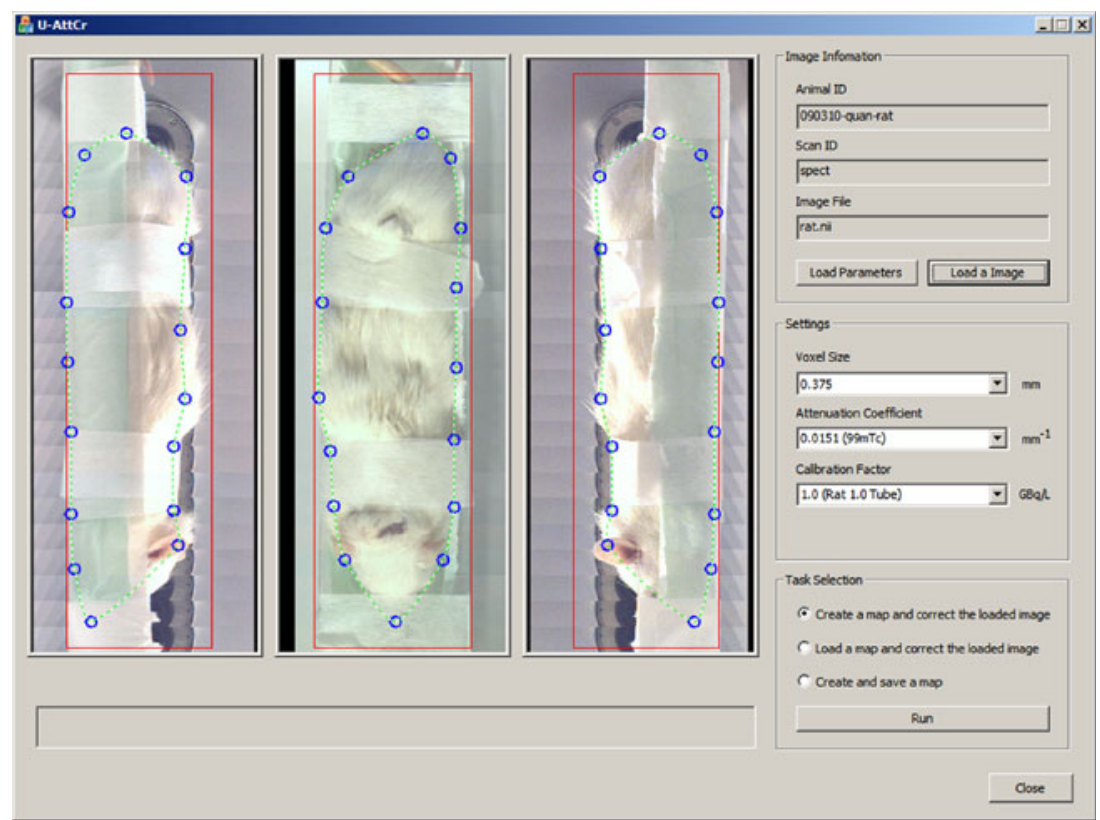

(a)

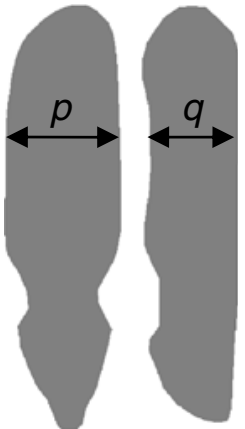

(b)

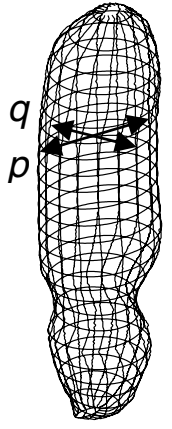

(c) 


\section{Experiments}

We first used a simple cylindrical phantom to validate the accuracy of absolute quantification with U-SPECT-II. The phantom (45 mm diameter and $40 \mathrm{~mm}$ height) was filled with ${ }^{99 \mathrm{~m}} \mathrm{Tc}$ solution with activity concentration equal to $2.88 \mathrm{MBq} / \mathrm{ml}$. The activity was measured by using the VIK202 dose calibrator. A scan was then performed with USPECT-II, and the image was subsequently reconstructed by using 6 POSEM iterations with 16 subsets, a $0.375-\mathrm{mm}$ voxel size, and with decay and scatter corrections integrated.

A cadaver of a $250 \mathrm{-g}$ female Wistar rat was used to test our method in a realistic complex attenuation distribution. Twelve ${ }^{99 \mathrm{~m}}$ Tc sphere-like sources were made from tips of microcentrifuge tubes (container), cotton balls (solution absorber), ${ }^{99 \mathrm{~m}} \mathrm{Tc}$ solution (radioactive source) and Parafilm (seal). Their diameters were around $5 \mathrm{~mm}$ and activities ranged from 7.29 to $9.87 \mathrm{MBq}$, measured in the same dose calibrator employed in the phantom experiment. These sources were inserted into the rat (mouth, neck, shoulder $\times$ 2 , lung $\times 2$, liver, right kidney, intestine, bladder and back $\times$ 2 ) by surgery. Then a total-body SPECT scan was carried out. The image was reconstructed by using 6 POSEM iterations combining with 16 subsets and a $0.375-\mathrm{mm}$ voxel size. Decay and scatter corrections were integrated into the reconstruction.

\section{Results}

\section{Phantom experiment}

Figure 3 shows reconstructed slices of the cylindrical phantom to illustrate the effect of the attenuation correction. The summation of ten transverse slices (decay corrected) without attenuation correction (Fig. 3a, b) is much darker than with attenuation correction (Fig. 3c, d), especially in the centre. This can be observed more clearly on the line profiles through the diameters of the phantom along the $X$ direction (Fig. 3e). The activity concentration measured in the dose calibrator as a gold standard is indicated by a horizontal line at $2.88 \mathrm{MBq} / \mathrm{ml}$.

Without corrections, the difference between the activity concentrations calculated on the reconstructed SPECT image and measured in the dose calibrator was significantly underestimated by $-0.54 \mathrm{MBq} / \mathrm{ml}$, or $-18.7 \%$. With scatter correction only, the difference worsened to $-0.77 \mathrm{MBq} / \mathrm{ml}$ $(-26.8 \%)$. Applying only attenuation correction led to an overestimation by $0.26 \mathrm{MBq} / \mathrm{ml}(9.2 \%)$. Applying attenuation correction in combination with scatter correction resulted in a small underestimation of $-0.05 \mathrm{MBq} / \mathrm{ml}(-1.7 \%)$. These numbers were calculated on the voxels in a cylindrical volume with a diameter of $42 \mathrm{~mm}$, which was slightly smaller than the

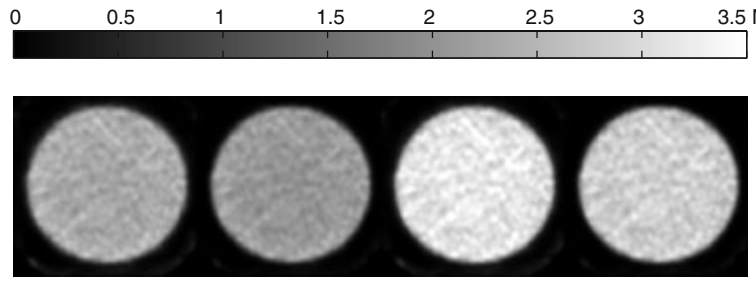

(a)

(b)

(c)

(d)

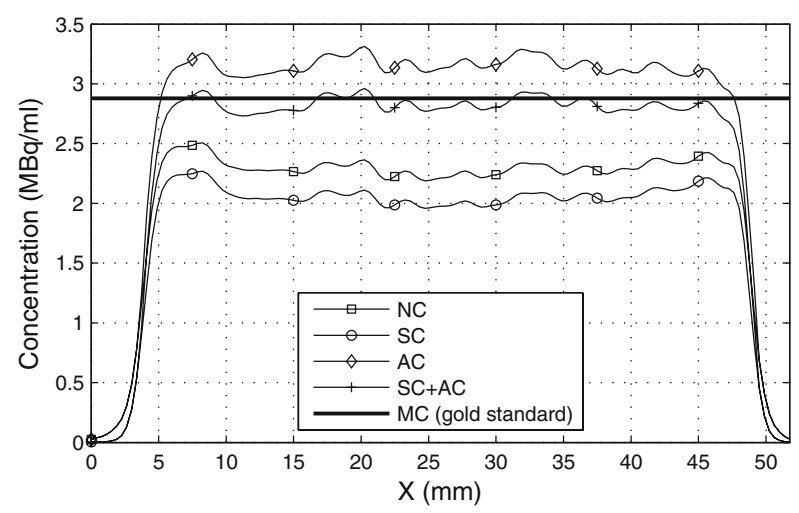

(e)

Fig. 3 Averages of ten transverse SPECT slices of the cylindrical phantom. The grey scales of $\mathbf{a}-\mathbf{d}$ are the same, from black $(0 \mathrm{Mbq} / \mathrm{ml})$ to white $(3.5 \mathrm{MBq} / \mathrm{ml})$. a Without corrections $(N C)$. b With scatter correction $(S C)$. $\mathbf{c}$ With attenuation correction $(A C)$. $\mathbf{d}$ With scatter and attenuation correction $(S C+A C)$. e Line profiles through centre of phantom. The line $M C$ indicates the concentration measured with a dose calibrator, as a gold standard

phantom size in order to avoid the influence of partial volume effect.

\section{Animal experiment}

Since the sources inserted into the rat were isolated well, it is possible to segment sub-volumes for individual sources on the reconstructed SPECT image. The activity of each source was calculated by adding all the voxel values in the sub-volume containing that source and then multiplying the resulting sum, the $C F$, and the voxel size together. Eleven volunteers were invited to carry out the attenuation corrections on the reconstructed image individually, using the application program that is shown in Fig. 2a. After a 15-min training session, all volunteers were able to finish their testing within 5 min. Table 2 lists the activities of the sources measured in the dose calibrator and their quantitative results calculated on the decay, scatter and attenuationcorrected image. The averages, standard deviations and percent errors of those results over the 11 testers are also listed.

Figure 4 shows the decay-corrected activities of the sources calculated on the reconstructed SPECT image without and with scatter and attenuation correction, as well as the activities measured in the dose calibrator. The 
Table 2 Activities of sources measured in dose calibrator and corrected results by 11 individual testers

\begin{tabular}{|c|c|c|c|c|c|c|c|c|c|c|c|c|c|c|c|}
\hline \multirow{2}{*}{$\begin{array}{l}\text { Source No. } \\
1\end{array}$} & \multirow{2}{*}{$\begin{array}{l}\begin{array}{l}\text { Activity } \\
\text { (MBq) }\end{array} \\
8.75\end{array}$} & \multicolumn{11}{|c|}{ Quantified results by 11 individual testers (MBq) } & \multirow{2}{*}{$\frac{\begin{array}{l}\text { Aver. } \\
(\mathrm{MBq})\end{array}}{8.68}$} & \multirow{2}{*}{$\begin{array}{l}\begin{array}{l}\mathrm{SD} \\
(\mathrm{MBq})\end{array} \\
0.21\end{array}$} & \multirow{2}{*}{$\begin{array}{l}\begin{array}{l}\text { Error } \\
(\%)\end{array} \\
-0.8\end{array}$} \\
\hline & & 8.91 & 8.78 & 9.00 & 8.43 & 8.86 & 8.54 & 8.69 & 8.69 & 8.50 & 8.31 & 8.80 & & & \\
\hline 2 & 8.77 & 7.92 & 8.25 & 8.23 & 7.82 & 8.09 & 8.23 & 8.38 & 8.02 & 8.51 & 8.18 & 8.87 & 8.23 & 0.29 & -6.2 \\
\hline 3 & 8.75 & 8.82 & 8.77 & 8.65 & 8.80 & 8.69 & 8.65 & 8.22 & 8.74 & 8.58 & 8.73 & 8.88 & 8.68 & 0.18 & -0.8 \\
\hline 4 & 7.87 & 8.07 & 8.19 & 8.13 & 8.25 & 8.27 & 7.96 & 8.34 & 8.09 & 8.04 & 8.33 & 8.15 & 8.16 & 0.12 & 3.7 \\
\hline 5 & 8.16 & 8.30 & 8.15 & 8.31 & 8.04 & 8.25 & 8.01 & 8.00 & 8.23 & 7.98 & 8.08 & 8.26 & 8.15 & 0.13 & -0.2 \\
\hline 6 & 7.94 & 8.21 & 7.94 & 7.78 & 7.94 & 8.03 & 7.63 & 7.52 & 8.02 & 7.76 & 7.92 & 7.97 & 7.88 & 0.19 & -0.7 \\
\hline 7 & 9.73 & 10.47 & 9.87 & 10.26 & 10.29 & 10.00 & 9.93 & 9.59 & 9.62 & 9.97 & 10.02 & 9.99 & 10.00 & 0.26 & 2.8 \\
\hline 8 & 7.29 & 7.82 & 7.59 & 7.82 & 7.78 & 7.68 & 7.60 & 7.45 & 7.31 & 7.63 & 7.68 & 7.65 & 7.64 & 0.15 & 4.8 \\
\hline 9 & 8.96 & 9.19 & 8.89 & 9.17 & 8.81 & 8.95 & 8.74 & 8.61 & 8.77 & 8.73 & 8.56 & 8.94 & 8.85 & 0.20 & -1.2 \\
\hline 10 & 9.27 & 9.63 & 9.47 & 9.68 & 9.39 & 9.49 & 9.32 & 9.29 & 9.40 & 9.29 & 9.17 & 9.53 & 9.42 & 0.15 & 1.7 \\
\hline 11 & 9.69 & 9.73 & 9.66 & 9.78 & 9.44 & 9.60 & 9.53 & 9.64 & 9.54 & 9.33 & 9.07 & 9.71 & 9.55 & 0.21 & -1.5 \\
\hline 12 & 9.87 & 9.91 & 9.88 & 10.02 & 9.64 & 9.97 & 9.62 & 9.72 & 9.84 & 9.62 & 9.60 & 9.85 & 9.79 & 0.15 & -0.8 \\
\hline Max. error & & & & & & & & & & & & & & & 6.2 \\
\hline
\end{tabular}

quantification errors on the uncorrected images ranged from -23.6 to $-9.3 \%$. With attenuation correction only, these errors of the 11 testers' average results ranged from -1.4 to $10.3 \%$, with an average magnitude of $5.6 \%$ over all 12 sources. With scatter and attenuation correction, these errors ranged from -6.3 to $+4.3 \%$, and the average magnitude decreased to $2.1 \%$.

\section{Discussion}

Quantification of SPECT imaging in absolute terms becomes possible when physical effects like collimator blurring, sensitivity, scatter and photon absorption are all modelled during iterative reconstruction or compensated afterwards. In clinical SPECT imaging, non-uniform attenuation maps (e.g. from X-ray CT) need to be acquired for accurate quantitative results [15]. Our work shows that attenuation compensation can be performed well in small-animal SPECT applications, despite the fact that no CT scanner was used. Uniform attenuation maps were created with the help of body contour information from optical cameras. At the current stage of this research, the 2-D contours were defined manually with deformable closed spline curves on the optical photos. This could possibly be further automated by using certain image processing techniques, such as image segmentation, pattern recognition and an anatomical body contour model.

Experiments were performed to validate our method, first with a simple uniform phantom, and then with a rat cadaver. Although the animal model of a rat with artificial sources is still different from a realistic set-up of pre-
Fig. 4 a Planar images showing positions of sources. $\mathbf{b}$ Activities of sources. $N C$ no correction was performed, $S C+A C$ scatter and attenuation correction was performed, $M A$ activities measured by dose calibrator

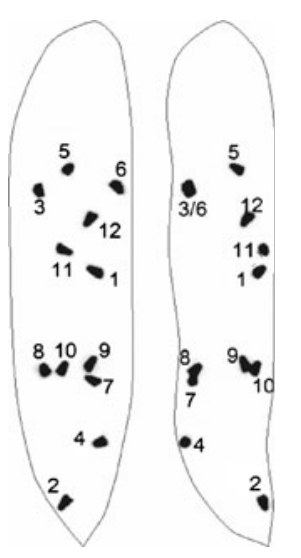

(a)

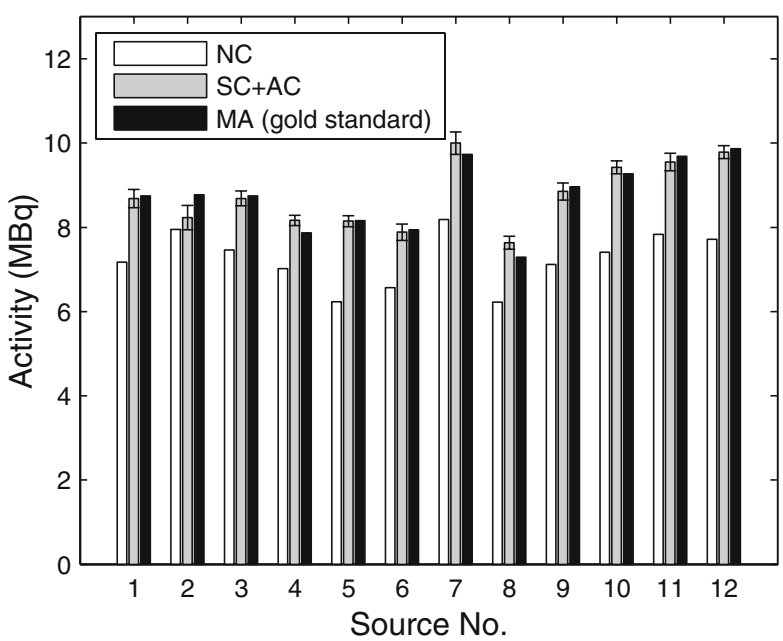

(b) 
clinical studies (with injections and region of interest measurements, etc.), it is very well suited for evaluating the accuracy of correction methods, since the exact amounts of activity in specific regions of interest are known (which is in contrast to using living animals with a tracer injected). The results from the phantom and animal experiments demonstrated that without compensation an approximately $10-30 \%$ underestimation of the activity concentration could be achieved, varying with the diameters of the objects and the depth of the sources. Note that even if the sources were just under the skin of the rat, i.e. the common positions of transplanted tumours for pre-clinical cancer research, there was still more than $10 \%$ underestimation. Applying a firstorder uniform attenuation correction with the Chang algorithm resulted in accurate quantifications in our experiments, especially when combining together with scatter correction $(-1.7 \%$ in the phantom study and from -6.3 to $+4.3 \%$ in the animal study).

In Table 2 we notice that the magnitudes of most errors are below 5\% except for source No. 2 which has an underestimation of $-6.3 \%$. This source was in the rat's mouth and we found that the mouth can be hardly seen on the optical photos, due to the obstruction of the tissue and tape around the rat, which makes the 2-D contours at that region uncertain. In fact, the source is not even enclosed with the contours made by 7 of the 11 testers. This apparently leads to an underestimation of the attenuation and thus to a negative bias of the quantitative result. Therefore, we suggest trying to keep a clear sight of the animal contour in a study that requires absolute quantification. On the other hand, the standard deviations of the 11 testers' results are small $(\leq 3 \%)$, which supports the observation that the proposed attenuation correction method is not very sensitive to the contour differences introduced by some subjective judgments of individuals.

When imaging with ${ }^{99 \mathrm{~m}} \mathrm{Tc}$, scatter correction is usually not performed in normal studies due to the small amount of Compton photons within the photopeak window. However, for absolute quantitative studies, it is better to apply scatter correction in order to avoid the overestimation caused by scattered photons. By using attenuation correction in combination with scatter correction, about 7.5 and 3.5\% improvement of quantitative accuracy over the accuracy with only attenuation correction was gained in our phantom and rat cadaver experiments, respectively.

There are two things in our experiments which may cause a bias to the results. The first one is the energy window settings of reconstruction. Different photopeak window settings will affect the proportion of gamma counts which contribute to the reconstructed images and thus change the calibration factor. To avoid this we employed the same window settings for all of the reconstructions. The other one is the inaccuracy of the dose calibrator. By using the same dose calibrator to measure the sources for computing the calibration factor and for the validation experiments, the system error, or bias, of the dose calibrator cancels out in the final results of relative errors. However, accurate calibration of the dose calibrator is essential to obtain the exact calibration factor and absolute quantitative results in applications.

In order to simplify our method and to facilitate rapid correction, the pinhole geometry of the U-SPECT-II system and associated attenuation paths were roughly (2-D) approximated during the Chang-like attenuation correction. The actual projection paths of a voxel are very complicated considering both the multi-pinhole geometry and the use of multiple bed positions during acquisition. It was shown that this approximation provides good quantitative accuracy in small-animal images. That still good results were obtained can partly be explained by the fact that changes of transmitted fraction due to the length differences between oblique paths and perpendicular paths are small (around 5\% at the most).

In clinical studies, the Chang algorithm is known to cause over- and undercorrection, and therefore an additional iterative step for compensation is implemented, however at the cost of noise increase. Since in small-animal SPECT the results are accurate without additional iterations, we restricted our method to pure post-reconstruction processing which (1) is much easier to implement and (2) does not increase noise.

\section{Conclusion}

The effects of attenuation in rat-sized objects are significant. We introduced a contour-based attenuation correction method for small-animal SPECT. To validate this method, phantom and animal experiments were performed and subsequently quantified with a practical software tool by 11 testers. From the results (average error of 1.7 and 2.1\% for phantom and animal studies, respectively), we conclude that this body contour-based uniform attenuation correction method derived from the Chang algorithm, in combination with scatter correction, is sufficient for accurate absolute quantification in small-animal SPECT imaging. The information of 3-D contours for generating the attenuation maps can be obtained from optical photos instead of from X-ray $\mathrm{CT}$ images. This gives opportunities to do absolute quantitative SPECT with stand-alone SPECT systems and to reduce the dose to the animals caused by X-rays which can be limiting in longitudinal studies.

Acknowledgments We thank Roel Wierts, Sergiy Lazarenko, Marcel Segbers, Jurgen Sijbesma, Norbert Gehéniau, and Paul Hermans for technical support, and Johan de Jong for suggestions and comments. 
Open Access This article is distributed under the terms of the Creative Commons Attribution Noncommercial License which permits any noncommercial use, distribution, and reproduction in any medium, provided the original author(s) and source are credited.

\section{References}

1. Jaszczak RJ, Li JY, Wang HL, Zalutsky MR, Coleman RE. Pinhole collimation for ultra-high-resolution, small-field-of-view SPECT. Phys Med Biol 1994;39:425-37.

2. McElroy DP, MacDonald LR, Beekman FJ, Wang YC, Patt BE, Iwanczyk JS, et al. Performance evaluation of A-SPECT: a high resolution desktop pinhole SPECT system for imaging small animals. IEEE Trans Nucl Sci 2002;49:2139-47. doi:10.1109/Tns.2002.803801.

3. Schramm NU, Ebel G, Engeland U, Schurrat T, Behe M, Behr TM. High-resolution SPECT using multipinhole collimation. IEEE Trans Nucl Sci 2003;50:315-20. doi:10.1109/Tns. 2003.812437.

4. Furenlid LR, Wilson DW, Chen YC, Kim H, Pietraski PJ, Crawford MJ, et al. FastSPECT II: a second-generation highresolution dynamic SPECT imager. IEEE Trans Nucl Sci 2004;51:631-5. doi:10.1109/Tns.2004.830975.

5. Beekman FJ, van der Have F, Vastenhouw B, van der Linden AJA, van Rijk PP, Burbach JPH, et al. U-SPECT-I: a novel system for submillimeter-resolution tomography with radiolabeled molecules in mice. J Nucl Med 2005;46:1194-200.

6. Metzler SD, Jaszczak RJ, Patil NH, Vemulapalli S, Akabani G, Chin BB. Molecular imaging of small animals with a triple-head SPECT system using pinhole collimation. IEEE Trans Med Imaging 2005;24:853-62. doi:10.1109/Tmi.2005.848357.

7. Hesterman JY, Kupinski MA, Furenlid LR, Wilson DW, Barrett $\mathrm{HH}$. The multi-module, multi-resolution system (M3R): a novel small-animal SPECT system. Med Phys 2007;34:987-93. doi:10.1118/1.2432071.

8. Rentmeester MCM, van der Have F, Beekman FJ. Optimizing multi-pinhole SPECT geometries using an analytical model. Phys Med Biol 2007;52:2567-81. doi:10.1088/0031-9155/52/9/016.

9. van der Have F, Vastenhouw B, Ramakers RM, Branderhorst W, Krah JO, Ji C, et al. U-SPECT-II: an ultra-high-resolution device for molecular small-animal imaging. J Nucl Med 2009;50:599_ 605. doi:10.2967/jnumed.108.056606.

10. Beekman F, van der Have F. The pinhole: gateway to ultra-highresolution three-dimensional radionuclide imaging. Eur J Nucl Med Mol Imaging 2007;34:151-61. doi:10.1007/s00259-006-0248-6.

11. Hwang AB, Franc BL, Gullberg GT, Hasegawa BH. Assessment of the sources of error affecting the quantitative accuracy of SPECT imaging in small animals. Phys Med Biol 2008;53:223352. doi:10.1088/0031-9155/53/9/002.

12. Beekman FJ, Kamphuis C, Hutton BF, van Rijk PP. Halffanbeam collimators combined with scanning point sources for simultaneous emission-transmission imaging. J Nucl Med 1998;39:1996-2003.

13. Kaplan MS, Haynor DR, Vija H. A differential attenuation method for simultaneous estimation of SPECT activity and attenuation distributions. IEEE Trans Nucl Sci 1999;46:535-41.

14. El Fakhri G, Buvat I, Benali H, Todd-Pokropek A, Di Paola R. Relative impact of scatter, collimator response, attenuation, and finite spatial resolution corrections in cardiac SPECT. J Nucl Med 2000;41:1400-8.

15. King M, Farncombe T. An overview of attenuation and scatter correction of planar and SPECT data for dosimetry studies. Cancer Biother Radiopharm 2003;18:181-90.

16. de Jong HWAM, Beekman FJ. Rapid SPECT simulation of downscatter in non-uniform media. Phys Med Biol 2001;46:621-35.
17. Beekman FJ, de Jong HWAM, van Geloven S. Efficient fully 3-D iterative SPECT reconstruction with Monte Carlo-based scatter compensation. IEEE Trans Med Imaging 2002;21:867-77. doi:10.1109/Tmi.2002.803130.

18. de Jong HW, Beekman FJ, Viergever MA, van Rijk PP. Simultaneous $(99 \mathrm{~m}) \mathrm{Tc} /(201) \mathrm{Tl}$ dual-isotope SPET with Monte Carlo-based down-scatter correction. Eur J Nucl Med Mol Imaging 2002;29:1063-71. doi:10.1007/s00259-002-0834-1.

19. de Wit TC, Xiao J, Nijsen JF, van het Schip FD, Staelens SG, van Rijk PP, et al. Hybrid scatter correction applied to quantitative holmium-166 SPECT. Phys Med Biol 2006;51:4773-87. doi:10.1088/0031-9155/51/19/004.

20. Xiao J, de Wit TC, Zbijewski W, Staelens SG, Beekman FJ. Evaluation of 3D Monte Carlo-based scatter correction for 201Tl cardiac perfusion SPECT. J Nucl Med 2007;48:637-44.

21. Schillaci O. Hybrid SPECT/CT: a new era for SPECT imaging? Eur J Nucl Med Mol Imaging 2005;32:521-4. doi:10.1007/ s00259-005-1760-9.

22. Cherry SR. Multimodality in vivo imaging systems: twice the power or double the trouble? Annu Rev Biomed Eng 2006;8:3562. doi:10.1146/annurev.bioeng.8.061505.095728.

23. Hwang $\mathrm{AB}$, Hasegawa $\mathrm{BH}$. Attenuation correction for small animal SPECT imaging using x-ray CT data. Med Phys 2005;32:2799-804.

24. Hwang AB, Taylor CC, VanBrocklin HF, Dae MW, Hasegawa BH. Attenuation correction of small animal SPECT images acquired with 125I-iodorotenone. IEEE Trans Nucl Sci 2006;53:1213-20.

25. Vanhove C, Defrise M, Bossuyt A, Lahoutte T. Improved quantification in single-pinhole and multiple-pinhole SPECT using micro-CT information. Eur J Nucl Med Mol Imaging 2009;36:1049-63. doi:10.1007/s00259-009-1062-8.

26. Chang LT. A method for attenuation correction in radionuclide computed tomography. IEEE Trans Nucl Sci 1978;25:638-43.

27. Beekman FJ, Vastenhouw B. Design and simulation of a highresolution stationary SPECT system for small animals. Phys Med Biol 2004;49:4579-92. doi:10.1088/0031-9155/49/19/009.

28. Vastenhouw B, van der Have F, van der Linden AJA, von Oerthel L, Booij J, Burbach JPH, et al. Movies of dopamine transporter occupancy with ultra-high resolution focusing pinhole SPECT. Mol Psychiatry 2007;12:984-7. doi:10.1038/sj.mp.4002028.

29. Blanckaert P, Burvenich I, Staelens S, De Bruyne S, Moerman L, Wyffels L, et al. Effect of cyclosporin A administration on the biodistribution and multipinhole muSPECT imaging of [123I] R91150 in rodent brain. Eur J Nucl Med Mol Imaging 2009;36:446-53. doi:10.1007/s00259-008-0968-x.

30. Vastenhouw B, Beekman F. Submillimeter total-body murine imaging with U-SPECT-I. J Nucl Med 2007;48:487-93.

31. Branderhorst W, Vastenhouw B, Beekman FJ. Pixel-based subsets for rapid multi-pinhole SPECT reconstruction. Phys Med Biol 2010;55:2023-34. doi:10.1088/0031-9155/55/7/015.

32. van der Have F, Vastenhouw B, Rentmeester M, Beekman FJ System calibration and statistical image reconstruction for ultrahigh resolution stationary pinhole SPECT. IEEE Trans Med Imaging 2008;27:960-71. doi:10.1109/TMI.2008.924644.

33. Ogawa K, Harata Y, Ichihara T, Kubo A, Hashimoto S. A practical method for position-dependent Compton-scatter correction in single photon emission CT. IEEE Trans Med Imaging 1991;10:408-12.

34. Bowsher JE, Johnson VE, Turkington TG, Jaszczak RJ, Floyd $\mathrm{CE}$, Coleman RE. Bayesian reconstruction and use of anatomical a priori information for emission tomography. IEEE Trans Med Imaging 1996;15:673-86.

35. van der Have F, Beekman FJ. Photon penetration and scatter in micro-pinhole imaging: a Monte Carlo investigation. Phys Med Biol 2004;49:1369-86.

36. van der Have F, Beekman FJ. Penetration, scatter and sensitivity in channel micro-pinholes for SPECT: a Monte Carlo investigation. IEEE Trans Nucl Sci 2006;53:2635-45. doi:10.1109/Tns. 2006.882739. 